\title{
Chemical study of the Chinese medicine Pi Han Yao
}

\author{
TENG PENG ${ }^{1}$, FURONG ZHAO ${ }^{1,2}$, XIAOYU CHEN ${ }^{1,3}$, GUIHUA JIANG $^{1}$ and SHAONAN WANG ${ }^{1,4}$ \\ ${ }^{1}$ College of Pharmacy, Chengdu University of Traditional Chinese Medicine, Chengdu, Sichuan 610075; \\ ${ }^{2}$ R\&D, Sichuan Meike Pharmaceutical Co., Ltd., Chengdu, Sichuan 610000; ${ }^{3}$ Department of Project, \\ Mianyang Fuzheng Chinese Herbology Development Co., Ltd., Chengdu, Sichuan 610016; ${ }^{4} \& D$, \\ Shijiazhuang Pharmaceutical Group Co., Ltd., Shijiazhuang, Hebei 050000, P.R. China
}

Received September 14, 2015; Accepted December 22, 2015

DOI: $10.3892 /$ br.2016.566

\begin{abstract}
The aim of the present study was to ivnestigate the chemical constituents of the Chinese medicine Pi Han Yao (Gueldenstaedtia delavayi Franch) decoction. Following this, the quantitative determination of the formononetin and maackiain content in Pi Han Yao was established. The chemical constituents were isolated by column chromatography and their structures were elucidated by analysis of spectrometric data and chemical evidence. High-performance liquid chromatography (HPLC) was used for the determination of the formononetin and maackiain content in Pi Han Yao. Seven flavanones were isolated from the Pi Han Yao decoction. Five of the chemical structures were elucidated as 1, 7,2'-dihydroxy-4'-methoxy-isoflavanol; 2, maackiain; 3, formononet in-7- $O$ - $\beta$-D-glucoside; 4 , formononetin; and $5,9-(\beta-\mathrm{D}-$-ribo furanosyl)-adenosine. The other two compounds and their structures require further study. Additionally, the linear range of formononetin and maackiain were 0.03992-0.3992 and $0.0292-0.292 \mu \mathrm{g}$, and their recoveries were 100.31 and $100.44 \%$. To the best of our knowledge, compounds 1-5 were obtained from Pi Han Yao for the first time. The HPLC method use for determination of formononetin and maackiain in Pi Han Yao was simple, accurate and reliable. Findings from the present study suggest that these methods may be used to evaluate the quality of Pi Han Yao and provide an experience basis for quality standards of this medicinal material.
\end{abstract}

\section{Introduction}

Pi Han Yao is a traditional Chinese herbal medicine, derived from the Gueldenstaedtia delavayi Franch plant (1). It was first termed 'Pi Han Yao' in the early 1970s in the Xichang Chinese

Correspondence to: Dr Guihua Jiang, College of Pharmacy, Chengdu University of Traditional Chinese Medicine, 37 Shierqiao Road, Chengdu, Sichuan 610075, P.R. China

E-mail: jiangguihua043014@163.com

Key words: Gueldenstaedtia delavayi Franch, chemical constituents, structural determination, quantitative determination, quality control
Herbal Medicine book (2). As a Panzhihua-Xichang region folk medicine, Pi Han Yao is mainly used to treat exogenous diseases and has been found to be safe and effective in treating fevers, headaches, dizziness, sore throat, gasping syndrome and cough. The whole dry plant is used, and is usually consumed in a decoction daily. Although there have been studies on the fat-soluble components of the Gueldenstaedtia delavayi Franch plant, the pharmacologically active mechanisms for the effectiveness of Pi Han Yao in treating exogenous diseases remain to be elucidated. In addition, the full chemical constituents of Pi Han Yao have yet to be elucidated and there have been no studies on the quantitative determination of the formononetin and maackiain content, which are essentially the active components of the plant. Therefore, the present study investigated the material basis and mechanisms of the Chinese medicine Pi Han Yao by determining the chemical constituents of the plant decoction. Seven flavanone compounds were isolated from the Pi Han Yao decoction. Based on spectrum analysis, 5 of the 7 were identified. The 5 identified chemical structures were 1, 7,2'-dihydroxy-4'-methoxy-isoflavanol; 2, maackiain; 3 , formononetin-7- $O-\beta$-D-glucoside; 4 , formononetin; and 5 , 9-( $\beta$-D-ribofuranosyl)-adenosine. To the best of our knowledge, this is the first time that these compounds have been isolated from Pi Han Yao. Further study is required to identify the other 2 compounds and establish their structures. Formononetin, 1 of the 7 compounds isolated from the Pi Han Yao water solution, has been shown to have estrogen-like effects $(3,4)$, antioxidation effects $(5)$, and it can stimulate the specific adaptive immune system $(5,6)$. This compound has also been reported to have beneficial effects for certain cancers, as well as antiatheroscloresis, and diuresis effects $(7,8)$. Maackiain also has a strong diuresis effect, and therefore, formononetin and maackiain were chosen as the index components in the quantitative measurement of Pi Han Yao (9).

\section{Materials and methods}

Plant materials. The plant material was identified as Gueldenstaedtia delavayi Franch by Professor Guihua Jiang.

Chemicals. The formononetin standard was purchased from Chengdu at Staples Biological Technology Co., Ltd. (Chendgu, China; batch no. 111703-201012). The maackiain standard was prepared by us to a purity of $99.5 \%$, as previously described (10). 
The chromogenic agent, $10 \%$ sulfuric acid ethanol and iodine steam, was purchased from Chengdu Kelon (Chengdu, China). Methanol and acetonitrile with chromatographic purification were from Fisher Scientific (Schwerte, Germany). Distilled water was used and all the reagents were of analytical grade.

Instrumentation and conditions. The following instruments were used in the study: Bruker Avance 600 probe: ${ }^{13} \mathrm{C}-{ }^{1} \mathrm{H}$ DUL TE: 300K (Bruker, Zurich, Switzerland); Zabspec (Micromass, Waters Corp., Manchester, UK); Rotary Evaporators RE-52 (Shanghai Yarong Biochemistry Instrument Plant, Shanghai, China); Ultraviolet Instrument ZF-2 (Shanghai Anting Electron Instrument Plant, Shanghai, China); high performance liquid chromatography (HPLC; Agilent 1200 HPLC; Agilent Technologies, Inc., Santa Clara, CA, USA); Dikma C18 chromatography column $(5 \mu \mathrm{m}$, 4.6x250 mm; Dikma, Beijing, China); and a X24 Digital display micro-melting point tester (Beijing Tech Instrument Co., Ltd., Beijing, China).

Silica gel column chromatography (200-300 mesh; Qingdao Oceanic Chemical Plant, Qingdao, China); high-performance thin-layer chromatography (HPTLC; Qingdao Oceanic Chemical Plant); thin-layer chromatography (TLC) silica gel G and silica gel GF254 (Qingdao Oceanic Chemical Plant); and Sephadex LH20 (Pharmacia, Uppsala, Sweden) were used.

Extraction and isolation. The whole dry plants of Pi Han Yao $(12 \mathrm{~kg})$ were extracted with 120 liters of water at $90^{\circ} \mathrm{C}$ three times, 30 min each time, and subsequently concentrated under vacuum to the 30 liters. Following this, the water extract was extracted with ethyl acetate (EtOAc) and n-butanol (n-BuOH), successively. The EtOAc and $\mathrm{n}-\mathrm{BuOH}$ layers were concentrated to 64.1 and $141.7 \mathrm{~g}$ of residues, respectively, and were subsequently separated by repeated chromatography yielding 7 flavanone compounds.

\section{Results}

Investigating the chemical constituent of Pi Han Yao Structural determination

Compound 1. White needles (methanol); melting point (mp), $195-197^{\circ} \mathrm{C} ;[\alpha]_{D}{ }^{20}+210^{\circ}$ (c 0.10 , methanol), exhibited a positive reaction to the ferric chloride-ferricyanatum kalium test. ${ }^{1} \mathrm{H}$-nuclear magnetic resonance (NMR): $\delta: 3.56$ (1H, m, H-3), 3.63 (3H, s, OCH3-4'), 3.78 (1H, t, J=10.26 Hz, H-2a), 4.29 (1H, dd, $\mathrm{J}=10.98,5.16 \mathrm{~Hz}, \mathrm{H}-2 \mathrm{a}), 5.62(1 \mathrm{H}, \mathrm{d}, \mathrm{J}=7.02 \mathrm{~Hz}, \mathrm{H}-4), 6.37(1 \mathrm{H}$, d, J=2.4 Hz, H-8), 6.56 (1H, dd, J=2.22, 8.04 Hz, H-5'), $6.67(1 \mathrm{H}$, d, J=2.22 Hz, H-3'), $6.86(1 \mathrm{H}, \mathrm{d}, \mathrm{J}=2.22 \mathrm{~Hz}, \mathrm{H}-8), 6.94(1 \mathrm{H}, \mathrm{dd}$, $\mathrm{J}=8.10,2.58 \mathrm{~Hz}, \mathrm{H}-6), 7.20(1 \mathrm{H}, \mathrm{d}, \mathrm{J}=8.40 \mathrm{~Hz}, \mathrm{H}-6$ '), $7.58(1 \mathrm{H}, \mathrm{d}$, $\mathrm{J}=8.40 \mathrm{~Hz}, \mathrm{H}-5) .{ }^{13} \mathrm{C}-\mathrm{NMR}$ : $\delta 162.6$ (C-4'), 8162.4 (C-2'), $\delta 161.5$ (C-7), $\delta 158.4$ (C-9), $\delta 133.8$ (C-5), $\delta 126.3$ (C-, 6'), $\delta 121.0$ (C-1'),

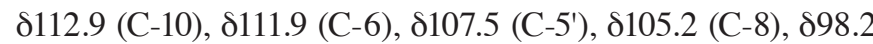
(C-3'), 880.2 (C-4), $\delta 67.7$ (C-2), 841.0 (C-3) and $\delta 56.3\left(\mathrm{C}-\mathrm{OCH}_{3}\right)$. Compound 1 was identified as 7,2'-dihydroxy-4'-methoxy-isoflavanol by comparison of Heteronuclear Single Quantum Correlation, H-H-Correlation Spectroscopy and Distortionless Enhancement by Polarisation Transfer data $(11,12)$.

Compound 2. Colorless needles (methanol, pyridine) reacted positively to $\mathrm{FeCl}_{3}$. The compound was purple-red when exposed to the UV lamp (254 nm), mp180-181 ${ }^{\circ} \mathrm{C}(12)$;
${ }^{1} \mathrm{H}-\mathrm{NMR}: 87.53$ (1H, d, J=8.82 Hz, H-1), 6.90 (1H, dd, J=8.46, $2.16 \mathrm{~Hz}, \mathrm{H}-2), 6.84$ (1H, s, H-7); $\delta 6.83$ (1H, d, J=2.22 Hz, H-4), 6.64 (1H, s, H-10), $5.91\left(1 \mathrm{H}, \mathrm{d}, \mathrm{J}=1.14 \mathrm{~Hz},-\mathrm{OCH}_{2} \mathrm{O}-\mathrm{aH}\right) ; 5.88$ $\left(1 \mathrm{H}, \mathrm{d}, \mathrm{J}=1.14 \mathrm{~Hz},-\mathrm{OCH}_{2} \mathrm{O}-\mathrm{bH}\right), 5.57(1 \mathrm{H}, \mathrm{d}, \mathrm{J}=7.32 \mathrm{~Hz}, \mathrm{H}-11 \mathrm{a}$, 4.25 (1H, dd, J=11.04, $4.74 \mathrm{~Hz}, \mathrm{H}-6 \beta), 3.77(1 \mathrm{H}, \mathrm{t}, \mathrm{J}=10.62 \mathrm{~Hz}$, H-6 $\alpha), 3.48$ (1H, m, H-6a). ${ }^{13}$ C-NMR: 840.5 (C-6a), 66.5 (C-6), 79.0 (C-11a), 93.7 (C-10), 101.5 (-OCH2-O), 104.0 (C-4), 105.3 (C-7), 110.7 (C-2), 111.8 (C-11b), 118.7 (C-6b), 132.5 (C-1), 141.9 (C-8), 148.3 (C-9), 154.7 (C-10a), 157.3 (C-4a), 160.4 (C-3). Mass spectrometry (MS): m/z: 307 [M+Na], m/z: 285 [M+H]. Compound 2 was identified as maackiain by comparison $\left({ }^{1} \mathrm{H}-\mathrm{NMR}\right.$ and $\left.{ }^{13} \mathrm{C}-\mathrm{NMR}\right)$ with previous data $(13,14)$.

Compound 3. White needles (methanol) that were easily soluble in methanol, mp217-219 ${ }^{\circ} \mathrm{C} .1 \mathrm{H}-\mathrm{NMR}$ : $\delta: 8.42(1 \mathrm{H}, \mathrm{s}$, H-2), 8.04 (1H, d, J=8.76 Hz, H-5), 7.52 (2H, d, J=8.40 Hz, H-2', 6'), 7.23 (1H, s, H-8), $7.13(1 \mathrm{H}, \mathrm{d}, \mathrm{J}=8.82 \mathrm{~Hz}, \mathrm{H}-6), 6.98(2 \mathrm{H}$, d, J=8.40 Hz, H-3', 5'), 5.09 (1H, d, J=6.96 Hz, glc H-1'), 3.30 $\left(3 \mathrm{H}, \mathrm{s}, \mathrm{OCH}_{3}\right)$. $84.5-5.5$ : Glycosidic bond $-\mathrm{OH}, \mathrm{\delta} 3-4$ glycosidic bond -H. ${ }^{13} \mathrm{C}-\mathrm{NMR}$ : $\delta 175.1$ (C-4), $\delta 161.9$ (C-7), $\delta 159.5$ (C-4'),

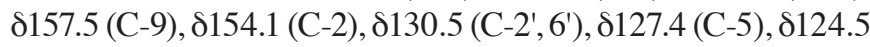

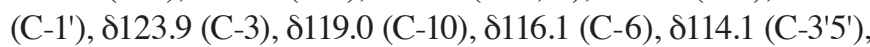
$\delta 103.9$ (C-8), $\delta 100.5$ (C-1'), 877.7 (C-3'), $\delta 77.0$ (C-5'), $\delta 73.6$ (C-2'), $870.1\left(\mathrm{C}-4^{\prime}\right), \delta 61.1(\mathrm{C}-6 '), \delta 55.6\left(\mathrm{C}^{\prime} \mathrm{OCH}_{3}\right)$. Compound 3 was identified as formononetin-7- $O-\beta-\mathrm{D}$-glucoside by comparison ( ${ }^{1} \mathrm{H}-\mathrm{NMR}$ and ${ }^{13} \mathrm{C}$-NMR) with previous data (15).

Compound 4 . White needles (methanol), mp257-258 ${ }^{\circ} \mathrm{C}$; showed a positive reaction to the ferric chloride-ferricyanatum kalium test. ${ }^{1} \mathrm{H}-\mathrm{NMR}$ : $\delta: 8.30(1 \mathrm{H}, \mathrm{s}, \mathrm{H}-2), 7.95(1 \mathrm{H}, \mathrm{dd}$, $\mathrm{J}=8.76,2.22 \mathrm{~Hz}, \mathrm{H}-5), 7.49$ (2H, d, J=8.40 Hz, H-2', 6'), 6.96 $\left(1 \mathrm{H}, \mathrm{d}, \mathrm{J}=8.76 \mathrm{~Hz}, \mathrm{H}-3^{\prime}, 5^{\prime}\right), 6.92$ (1H, dd, J=8.76, $\left.2.22 \mathrm{~Hz}, \mathrm{H}-6\right)$, 6.85 (2H, d, J=2.22 Hz, H-8), 3.77 (3H, s, OCH3). ${ }^{13} \mathrm{C}-\mathrm{NMR}$ :

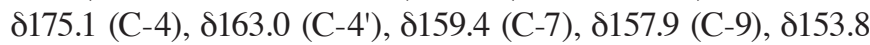
(C-3), $\delta 130.5$ (C-2', 6'), $\delta 127.8$ (C-5), $\delta 124.7$ (C-1'), 1123.6 (C-2),

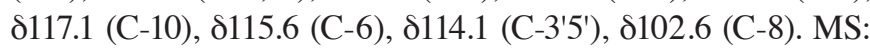
m/z: $291[\mathrm{M}+\mathrm{Na}], \mathrm{m} / \mathrm{z}: 269[\mathrm{M}+\mathrm{H}]$. Compound 4 was identified as formononetin by comparison with previous data $(11,16)$.

Compound 5. White needles (methanol), soluble in dimethyl sulfoxide; ${ }^{1} \mathrm{H}-\mathrm{NMR}$ : $\delta: 8.32$ (1H, s, H-8), 8.12 (1H, s, H-2), 7.30 (2H, s, H-NH2), 5.86 (1H, d, J=6.24 Hz, H-1'), 5.00-5.50 (3H, OH-H), 4.59 (1H, m, H-2'), 4.12 (1H, m, H-3'), 3.94 (1H, m, H-4'), 3.65 (1H, m, H-5'), 3.53 (1H, m, H-5'). ${ }^{13} \mathrm{C}-\mathrm{NMR}$ : $\delta 156.6$ (C-6), $\delta 152.8$ (C-2), $\delta 149.6$ (C-4), $\delta 140.4$ (C-8), $\delta 119.8$ (C-5), $\delta 88.4$ (C-1'), $\delta 86.4$ (C-4'), $\delta 73.9$ (C-2'), 871.1 (C-3'), $\delta 62.2$ (C-5'). MS: $\mathrm{m} / \mathrm{z}: 255[\mathrm{M}+\mathrm{Na}], \mathrm{m} / \mathrm{z}: 237[\mathrm{M}+\mathrm{H}]$. Compound 5 was identified as 9-( $\beta$-D-ribofuranosyl)-adenosine by comparison $\left({ }^{1} \mathrm{H}-\mathrm{NMR}\right.$ and $\left.{ }^{13} \mathrm{C}-\mathrm{NMR}\right)$ with literature data $(17,18)$.

The structures of compounds 1-5 are shown in Fig. 1. The structures of compounds 6 and 7 were complex and thus remain to be identified.

Content tests of formononetin and maackiain in Pi Han Yao Chromatographic conditions. The detection of the compounds was performed at $310 \mathrm{~nm}$. Satisfactory separation was obtained with a reverse-phase analytical column $(250 \times 4.6 \mathrm{~mm}, 5 \mu 1$, serial no. 8132964) and eluted with a methanol:water solution $(70: 30, \mathrm{v} / \mathrm{v})$ at a flow rate of $1.0 \mathrm{ml} / \mathrm{min}$. The column temperature was $25^{\circ} \mathrm{C}$ and the injection volume was $10 \mu l$. Theoretical plate numbers of formononetin and maackiain were $>4,000$.

Standard solutions. i) Preparation of stock standard solutions: Formononetin and maackiain stock solution were 
Table I. Calibration graphs for the Pi Han Yao constituents.

\begin{tabular}{lll}
\hline Constituent & Regression equation $\left(\mathrm{R}^{2}\right)$ & Linear range, $\mu \mathrm{g}$ \\
\hline Formononetin & $\mathrm{y}=4535.1 \mathrm{x}+18.587(0.9995)$ & $0.03992-0.3992$ \\
Maackiain & $\mathrm{y}=4258.3 \mathrm{x}+774.11(0.9995)$ & $0.0292-0.292$ \\
\hline
\end{tabular}

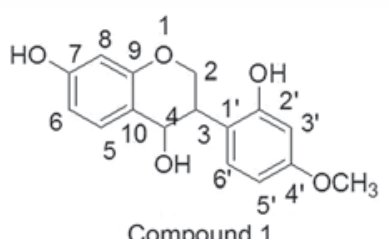

7, 2'dihydroxy-4'-methoxy-isoflavanol

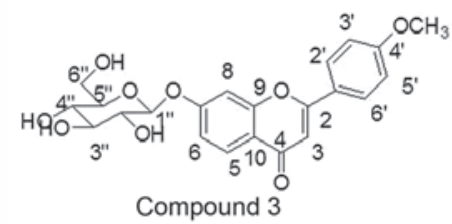

Formononetin-7-O- $\beta$-D-glucoside

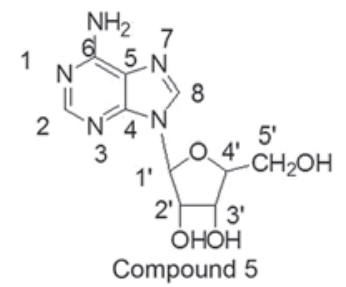

9-( $\beta$-D-ribofuranosyl)-adenosine

Figure 1. Structure of Pi Han Yao constituents.

prepared to a final concentration of $0.73 \mathrm{mg} / \mathrm{ml}$ in methanol. Chromatograms are shown in Fig. 2A and B.

ii) Sample preparation. The Pi Han Yao powder (3 g) was processed with ultrasonic extraction (power, $300 \mathrm{~W}$; rate, $50 \mathrm{~Hz}$ ) for $1 \mathrm{~h}$ in EtOAc, and subsequently recovered EtOAc in vacuo to yield the extract. The extract was dissolved with methanol in a
A

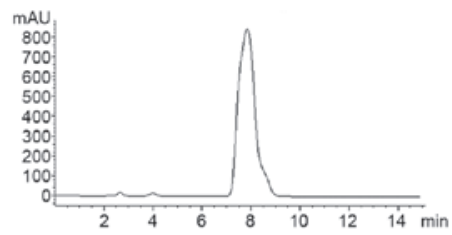

B

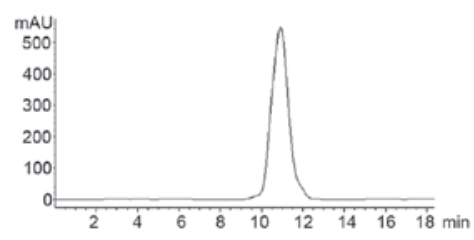

C

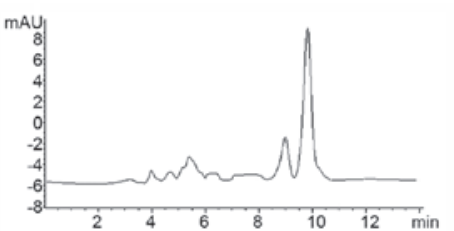

Figure 2. Chromatograms of (A) maackiain; (B) formononetin; and (C) Pi Han Yao.

$10 \mathrm{ml}$ volumetric flask and filtered through a $0.45-\mu \mathrm{m}$ filter prior to injection. The chromatogram is shown in Fig. 2C.

Calibration. Calibration curves were constructed in the range of 2-20 $\mu \mathrm{l}$ for formononetin and maackiain. The regression equation curves are listed in Table I.

The data showed a linear association between peak areas and concentration over the listed range for formononetin and maackiain. The detection limit was 0.03992-0.3992 $\mu \mathrm{g}$ for formononetin and 0.0292-0.292 $\mu \mathrm{g}$ for maackiain.

Precision and accuracy. The precision of injection was evaluated by performing replicate injections of the sample solution, and the relative standard deviations of formononetin and maackiain were 0.26 and $0.55 \%$, respectively. These results demonstrated that the method was highly precise.

Stability. To confirm that the standards remained stable over time, the concentrations of the two components were determined at $0,2,4,8,12$ and $24 \mathrm{~h}$, respectively, and the solution remained relatively stable. The RSD values of formononetin and maackiain were maintained at 1.59 and $2.57 \%$, respectively. The stability of the sample solutions was also evaluated

Table II. Results of the quantitative determination of the Pi Han Yao samples $(n=10)$.

\begin{tabular}{|c|c|c|c|c|c|c|}
\hline \multirow[b]{2}{*}{ Sample } & \multirow[b]{2}{*}{ Producing area in China } & \multirow[b]{2}{*}{ Growth } & \multicolumn{2}{|c|}{ Formononetin } & \multicolumn{2}{|c|}{ Maackiain } \\
\hline & & & Peak area & Content, $\mu \mathrm{g} / \mathrm{g}$ & Peak area & Content, $\mu \mathrm{g} / \mathrm{g}$ \\
\hline 1 & Te Erguo, $\mathrm{Pu} \mathrm{Ge}$ & Wild & 750.9 & 0.651 & 351.3 & 0.0211 \\
\hline 2 & Happy, Man Shuiwan, Mian Ning & Wild & 853.9 & 0.741 & 339.6 & 0.0204 \\
\hline 3 & Dawn, Sha Ba, Mian Ning & Cultivated & 660.4 & 0.573 & 206.8 & 0.0124 \\
\hline 4 & Victory, Sha Ba, Mian Ning & Wild & 867.7 & 0.753 & 368.5 & 0.0212 \\
\hline 5 & Li Zheng, Hong Mo, Mian Ning & Wild & 879.0 & 0.762 & 476.3 & 0.0286 \\
\hline 6 & Long Feng, Sha Ba, Mian Ning & Wild & 711.8 & 0.617 & 481.7 & 0.0289 \\
\hline 7 & Shi an, Da Xing & Cultivated & 664.3 & 0.576 & 382.1 & 0.0229 \\
\hline 8 & Min Yun, Xi Ning & Wild & 719.6 & 0.624 & 421.8 & 0.0253 \\
\hline 9 & Lang Huan, Lang Huan & Cultivated & 728.0 & 0.631 & 318.3 & 0.0191 \\
\hline 10 & A Yue, De Chang & Cultivated & 709.8 & 0.616 & 369.0 & 0.0222 \\
\hline
\end{tabular}


in the same manner by determining the concentrations of the two components at $0,2,4,8$ and $12 \mathrm{~h}$, respectively. The test solution remained relatively stable. The RSD values of formononetin and maackiain were maintained at 2.18 and $5.98 \%$, respectively.

Recovery study. The percentage of recovery was determined by adding a known concentration of the formononetin and maackiain standards to selected samples during the extraction process. The amounts added were $\sim 50 \%$ of the actual concentration of the samples. The concentration of the formononetin and the maackiain standards in the mixture were subsequently determined using the same methods described for the sample analysis. Without exception, recovery rates of $100.31 \%$ were achieved for the compound analyzed.

Quantitative determination of samples. The formononetin and maackiain contents of Pi Han Yao from plants collected from 10 different sources were determined in the same way as the sample analysis. The results are shown in Table II.

Analysis of the different Pi Han Yao plants from the 10 locations showed that the contents of formononetin and maackiain varied depending on the source. The content of formononetin ranged from $0.576-0.762 \mu \mathrm{g} / \mathrm{g}$ with an average value of $0.6544 \mu \mathrm{g} / \mathrm{g}$. The content of maackiain ranged from $0.0124-0.0289 \mu \mathrm{g} / \mathrm{g}$, with an average value of $0.02221 \mu \mathrm{g} / \mathrm{g}$. Overall the content of the two chemicals in wild Pi Han Yao was generally higher compared to cultivated plants. However, the difference was not significant. Furthermore, the content of formononetin and maackiain in Pi Han Yao was lower compared to the plant itself.

\section{Discussion}

To the best of our knowledge, the chemical constituents of the Chinese medicine Pi Han Yao decoction were investigated for the first time in this study. Five flavanone compounds were identified as follows: 1, 7,2'-dihydroxy-4'-methoxy-isoflavanol; 2 , maackiain; 3 , formononetin-7- $O-\beta$-D-glucoside; 4 , formononetin; and 5, 9-( $\beta$-D-ribofuranosyl)-adenosine. These compounds were obtained from Pi Han Yao for the first time, in accordance with previous studies (19-24).

The result of the formononetin and maackiain contents of Pi Han Yao from 10 different areas is shown in Table II. A method to determine formononetin and maackiain in the Pi Han Yao by HPLC has been reported in the present study, and this method is convenient, efficient, accurate, reliable and provides good replications, and thus, can be used for the quality control of Pi Han Yao.

The formononetin and maackiain contents were scanned from 200 to $400 \mathrm{~nm}$ by UV-1100. The maximum absorption wavelength of formononetin was $250 \mathrm{~nm}$, and the maximum absorption wavelengths of maackiain were 310 and $215 \mathrm{~nm}$. In order to determine the formononetin and maackiain content at the same conditions, the detection at $310 \mathrm{~nm}$ exhibited high sensitivity and little interference. Therefore, formononetin and maackiain were detected at $310 \mathrm{~nm}$.

In conclusion, the present study examined the extraction efficiency in solvents, the extraction method and the extraction time. The results showed that ethyl acetate ultrasound extraction had the highest extraction efficiency for constituents of Pi Han Yao.

\section{References}

1. Editorial Committee of the Flora of China of Chinese Academy of Science. Flora of China. Science Press, Beijing, 2004.

2. Province XChbiSC: Xi Chang Chinese Herbal Medicine (Vol 1). Xi Chang People's Enterprise, Xi Chang, 1972.

3. Wang WQ, Han ZK, Chen WH and Chen J: The enhancing effect of formononetin on the development of mammary gland in mice and exploration of its mechanism. Journal of Nanjing Agricultural University 16: 19, 1993.

4. Xin D, Liu Z, Xue C, et al: Study on estrogen-like effects of formononetin and its correlation with blood lipids. Chinese Journal of Gerontology 29: 2340, 2009.

5. Fu N, Liu C, Zhang R, et al: Study on the antioxidation about the flavonoids and triterpene compounds of glycyrrhiza. Pharmacology and Clinics of Chinese Materia Medica 5: 26, 1994.

6. Zhang $\mathrm{R}$ and Zhengkang $\mathrm{H}$ : Explore the effect of flavonoids on immunologic function in mice and $\beta$-endorphin level in the blood of mice. Chinese Journal of Immunology 10: 177, 1994.

7. Qu X and Jingdao L: Summarized the recent developments about the flavonoids of Maackia amurensis Rupr. et. Maxim. Journal of Medical Science Yan Bian University 16: 231, 1993.

8. Roberts DW, Doerge DR, Churchwell MI, Gamboa da Costa G, Marques MM and Tolleson WH: Inhibition of extrahepatic human cytochromes P450 1A1 and 1B1 by metabolism of isoflavones found in Trifolium pratense (red clover). J Agric Food Chem 52: 6623-6632, 2004.

9. Miao K, Zhang J, Dong Y, et al: The advances in the study on chemical components and pharmacological action of Sophora flavescens. Natural Product Research and Development 13: 69-73, 2001.

10. Wang $\mathrm{CH}$, Wang Y, Wang GC, et al: Chemical constituents from roots of Millettia speciosa. Chinese Traditional and Herbal Drugs 39: 972-975, 2008 (In Chinese).

11. Li HN, Yan H, Pang XY, et al: Study on chemical constituents of flavonoids of Sophora tonkinensis. China Journal of Chinese Materia Medica 349: 282-285, 2009.

12. Gomotsang B, Cornelius CWW, Runner RTR, et al: Two new isoflavanoids from Bolusanthus speciosus. Bulletin of the Chemical Society of Ethiopia 15: 131, 2001.

13. Gao D and Ruyi Z: Study on chemical constituents of glycyrhiza yunnanensis from Yunnan province. Chinese Herbal Medicines 25: 507-508, 1994.

14. Wu Li Jun, Toshio Miyase, Akira Ueno, et al: Studies on the constituents of Sophora flavesens AITON.II. Chemical and Pharmaceutical Bulletin 33: 3231-3236, 1985.

15. Cui BL, Nakamura M, Kinjo J, et al: Chemical Constituents of Astragali Semen. Chemical and Pharmaceutical Bulletin 41: 178-182, 1993.

16. Song C, Zheng ZLD, et al: Study on the isoflavone compounds of Astragalus membranaceus (Fisch.) Bunge. Chinese Bulletin of Botany 39: 764, 1997.

17. Basílio Janke EM, Limbach HH and Weisz K: Binding of an acetic acid ligand to adenosine: A low-temperature NMR study. J Am Chem Soc 126: 2135-2141, 2004.

18. Chenon MT, Pugmire RJ, Grant DM, Panzica RP and Townsend LB: Carbon-13 magnetic resonance. XXV. A basic set of parameters for the investigation of tautomerism im purines. Established from carbon-13 magnetic resonance studies using certain purines and pyrrolo [2,3-d]pyrimidines. J Am Chem Soc 97: 4627-4636, 1975.

19. Han Y, Gao L and Taibao W: Study on chemical components of essential oil of gueldenstaedtia multiflora bge. Journal of Shanxi College of TCM 11: 18-21, 2010.

20. Han Y and Liming G: Study on chemical constituents of gueldenstaedtia multiflora bge. Journal of Shanxi College of TCM 10: 83-84, 2009.

21. Li K, Li XM and Wang BG: Chemical from herd of constituents of Gueldenstaedtia stenophylla. Zhongguo Zhong Yao Za Zhi 33: 1711-1713, 2008 (In Chinese).

22. Wang $\mathbf{J}$ and Rong Z: Study on the chemical constituents of Gueldenstaedtia multiflora Bge. Acta Botanica Boreali-Occidentalia Sinica 9: 127-130, 1989.

23. Wei YX, Chen L and Wang JX: Studies on the chemical constituents of Gueldenstaedtia stenophylla. Zhong Yao Cai 30: 954-956, 2007 (In Chinese).

24. Zhu Rong ZD and Xu RS: Study on the chemical constituents of Gueldenstaedtia multiflora Bge. Chinese Traditional and Herbal Drugs 15: 1-3, 1984. 